\title{
Ischemic Preconditioning Attenuates Portal Venous Plasma Concentrations of Purines following Warm Liver Ischemia in Man
}

\author{
A. Choukèr ${ }^{a}$ A. Martignoni ${ }^{a}$ R.J. Schauer ${ }^{b}$ H.G. Rau ${ }^{b} \quad$ A. Volk ${ }^{b}$

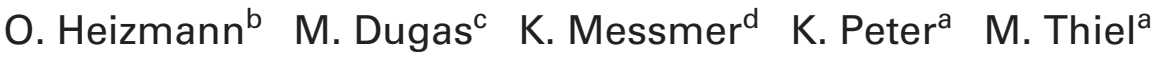 \\ ${ }^{a}$ Clinic of Anesthesiology and ${ }^{b}$ Department of Surgery, ${ }^{c}$ Institute for Epidemiology and Biometry, \\ ${ }^{d}$ Institute for Surgical Research, Klinikum Grosshadern, Ludwig Maximilians University, Munich, Germany
}

\section{Key Words}

Adenine nucleotides - Preconditioning - Reperfusion • Hepatectomy

\begin{abstract}
Background/Aims: Degradation of adenine nucleotides to adenosine has been suggested to play a critical role in ischemic preconditioning (IPC). Thus, we questioned in patients undergoing partial hepatectomy whether (i) IPC will increase plasma purine catabolites and whether (ii) formation of purines in response to vascular clamping (Pringle maneuver) can be attenuated by prior IPC. Methods: 75 patients were randomly assigned to three groups: group I underwent hepatectomy without vascular clamping; group II was subjected to the Pringle maneuver during resection, and group III was preconditioned (10 min ischemia and $10 \mathrm{~min}$ reperfusion) prior to the Pringle maneuver for resection. Central, portal venous and arterial plasma concentrations of adenosine, inosine, hypoxanthine and xanthine were determined by high-performance liquid chromatography. Results: Duration of the Pringle maneuver did not differ between patients with or without IPC. Surgery without vascular clamping had only a minor effect on plasma purine concentrations. After IPC, plasma concentrations of purines
\end{abstract}

transiently increased. After the Pringle maneuver alone, purine plasma concentrations were most increased. This strong rise in plasma purines caused by the Pringle maneuver, however, was significantly attenuated by IPC. When portal venous minus arterial concentration difference was calculated for inosine or hypoxanthine, the respective differences became positive in patients subjected to the Pringle maneuver and were completely prevented by preconditioning. Conclusion: These data demonstrate that (i) IPC increases formation of adenosine, and that (ii) the unwanted degradation of adenine nucleotides to purines caused by the Pringle maneuver can be attenuated by IPC. Because IPC also induces a decrease of portal venous minus arterial purine plasma concentration differences, IPC might possibly decrease disturbances in the energy metabolism in the intestine as well.

Copyright $\odot 2005$ S. Karger AG, Basel

\section{Introduction}

Ischemic preconditioning (IPC) has been shown to be a powerful protective measure in different hepatic models of warm [1] and cold [2] ischemia-reperfusion injury. Although protection from ischemic injury by ischemic pre-

\section{KARGER}

Fax +4161306 1234

E-Mail karger@karger.ch

www.karger.com
(C) 2005 S. Karger AG, Basel

0014-312X/05/0373-0144\$22.00/0

Accessible online at: www.karger.com/esr
Manfred Thiel, MD

Clinic of Anesthesiology, Klinikum Grosshadern

Marchioninistrasse 15, DE-81377 Munich (Germany)

Tel. +49 897095 3402, Fax +49 8970958886

E-Mailmthiel@med.uni-muenchen.de 


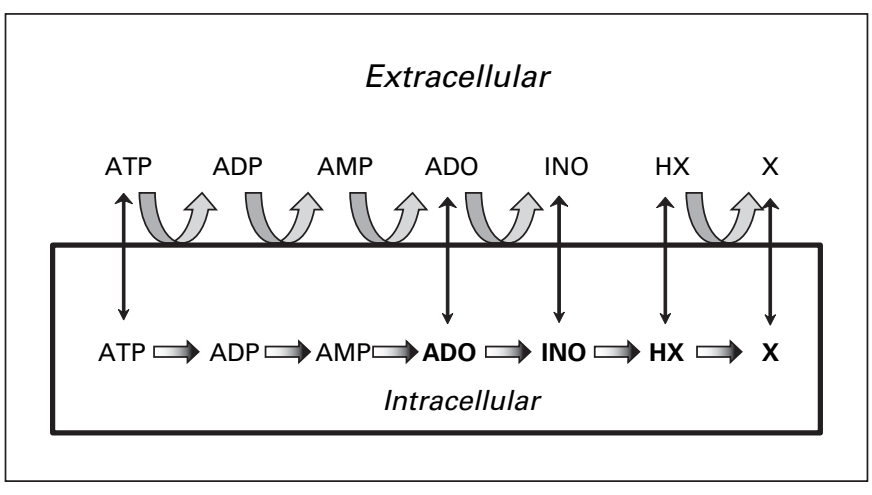

Fig. 1. Adenine nucleotides and nucleoside-related intracellular and extracellular exchange and metabolic pathways. ATP = Adenosine triphosphate; ADP = adenosine diphosphate; $\mathrm{AMP}=$ adenosine monophosphate; $\mathrm{ADO}=$ adenosine; $\mathrm{INO}=$ inosine; HX = hypoxanthine; $\mathrm{X}=$ xanthine [22].

conditiong is well documented, the underlying mechanisms have not been identified so far. Among many others (catecholamines, kinins, opioids, eicosanoids and nitric oxide (NO)), adenosine has been suggested to be a central mediator in hepatic preconditioning [3]. Even short periods of ischemia cause the enhanced breakdown of adenine nucleotides to adenosine (fig. 1). Although hypoxia predominantly results in the degradation of intracellular adenine nucleotides, adenine nucleotides can also be released into the extracellular space via specific transporters or e.g. by the reperfusion-induced damage of cell membranes. In the extracellular space, adenosine triphosphate (ATP) is rapidly hydrolyzed by a cascade of ectonucleotidases to generate adenosine and its degradation product inosine again. Both intracellular and extracellular adenosine are in steady state across the cell membrane via high- and low-capacity nucleoside transporters.

Irrespective of its sites of generation, adenosine can bind to extracellular specific receptors. Acting via $\mathrm{A}_{2}$ receptors on hepatocytes [3] and sinusoidal endothelial cells [2], adenosine was suggested to increase the formation of $\mathrm{NO}$ as a more downstream located mediator of IPC [1]. The half-life of adenosine in plasma, which is similar to $\mathrm{NO}$, is very short. This half-life is $<2 \mathrm{~s}$ at its concentration in the normal range (up to $150 \mathrm{nM}$ ) [4].

Under physiological conditions, removal of adenosine from plasma occurs via the uptake by erythrocytes and vascular endothelial cells through the aforementioned high- and low-capacity nucleoside transporters.

Under pathological conditions, e.g. hypoxia, adenosine is predominantly degraded by the enzyme adenosine deaminase to inosine. Since adenosine deaminase is also present in the plasma, deamination of adenosine to inosine there also occurs. Like adenosine, extracellular inosine can be taken up by cells through the transmembranal nucleoside transporters. Together with the inosine already formed in the cell, inosine is further degraded to hypoxanthine and the latter to xanthine. Thus, in addition to direct determination of adenosine, measurement of the purine metabolites inosine, hypoxanthine and xanthine indirectly reflects its formation and approximately equals the degradation of high-energy adenine nucleotides during hepatic ischemia.

Regarding the pathophysiological role of purines during ischemia-reperfusion, we hypothesized that (i) IPC will induce formation of adenosine and (ii) IPC will attenuate adverse metabolic effects caused by prolonged periods of ischemia (e.g. Pringle maneuver for liver resection). Accordingly, we questioned in patients undergoing partial hepatectomy (i) whether even a brief period of IPC will be sufficient to cause a transient increase of plasma purines, especially of hepato-protective adenosine and (ii) whether the Pringle maneuver induced unwanted degradation of adenine nucleotides to purines can be attenuated by IPC.

\section{Material and Methods}

\section{Study Design and Randomization}

The study protocol was approved in accordance with the guidelines of the 1975 Declaration of Helsinki by the Ethics Committee of Ludwig Maximilians University of Munich and written informed consent was obtained from each patient before surgery. 100 patients were tested for eligibility from March 1999 to August 2000. One subject was excluded from randomization, because he did not meet the inclusion criteria which were: (1) an American Society of Anesthesiologists' Physical Status (ASA-PS) - classification score 4 or 5 ; (2) other than hepatic tumor was diagnosed unexpectedly before surgery; (3) severe liver steatosis or cirrhosis was assumed prior to surgery, and (4) when patient's age was $<18$ years. After enrolment and evaluation by the participating physicians, the patients were assigned by lot to one of the three study groups. For that purpose, three lots were labeled with NPR, PR and IPC, were folded thereafter and then placed into a bowl. One lot was taken out of three. For the next patient the same procedure was repeated with three new lots. Hence, a total of 99 patients were allocated to NPR $(n=28)$ to PR $(n=33)$ and to IPC $(n=38)$. Intraoperatively, a few patients could not receive the procedure which was designated by lot, because of surgical reasons. Therefore, 2 patients from the NPR group were cross-allocated to the PR group, while 3 patients from the PR group and 2 patients from the IPC group were cross-allocated to the NPR group. In addition, patients had to be excluded intraoperatively for reasons of drug administration (anti-inflammatory drugs, e.g. pentoxfylline, corticosteroids) or when a liver cirrhosis was intraoperatively identified. Moreover, other surgical rea- 
sons (inoperability, massive hemorrhage, additional surgical procedures, e.g. bilio-enteric anastomosis) led to further dropouts. Thus, of 99 patients randomly assigned to three study groups, 25 patients in each group underwent elective major hepatectomy (for further details also referring to CONSORT criteria, see Choukèr et al. [16]). Because of difficult blood collection from the portal vein and the loss of a few samples due to inappropriate handling, the number of specimens available for analyses was smaller than 25 per group.

In group I, partial hepatectomy was performed without vascular clamping of the portal triad (Pringle maneuver). In group II, patients were subjected to the Pringle maneuver during parenchymal resection. In group III, livers of patients were preconditioned by a brief period of $10 \mathrm{~min}$ of ischemia followed by $10 \mathrm{~min}$ of reperfusion prior to the Pringle maneuver.

\section{Anesthesia and Surgery}

All operations were performed under general anesthesia (thiopental, cis-atracurium, fentanyl, desflurane 5-6 vol\%) in combination with epidural anesthesia according to a standardized protocol. Body temperature was kept between 36.0 and $37.0^{\circ} \mathrm{C}$ by continuous warming with a convective air-warming system.

All patients underwent laparotomy. For right hemihepatectomies the liver was mobilized to expose the inferior vena cava and the short caudate veins were ligated and divided before portal triad clamping. Similarly, hepatic veins were isolated, but not ligated prior to the IPC or Pringle maneuver. Clamping of the portal triad was performed without affecting the bile duct. Transection was started immediately after inducing the Pringle maneuver which was maintained until the transection was finished. Parenchymal transection was performed by use of a water jet cutter (Saphir Medical, Lyon, France) [6]. The volume of the resected liver was determined by the quantity of displaced fluid in a prefilled trough. Operations were performed by staff surgeons.

\section{Plasma Concentrations of Purines and Lactate}

Central venous and arterial blood samples were drawn through indwelling catheters. Insertion of central venous catheters was controlled by ECG tracing in order to place tips of catheters in the right atrium. Portal venous blood samples were drawn intraoperatively by direct puncture of the portal vein. Plasma concentrations of purine nucleosides (adenosine and inosine) were analyzed by dual column switching high-affinity performance/reversed-phase highperformance liquid chromatography technique using an internal surface nitrophenylboronic acid precolumn as previously described [7]. Purine bases (hypoxanthine and xanthine) were determined by reversed-phase high-performance liquid chromatography by a modified method published previously [8]. Plasma lactate concentrations were amperiometrically determined by the lactase oxidase catalyzed oxidation reaction using a blood gas analyzer (ABL 700, Radiometer, Copenhagen, Denmark).

\section{Hepatocellular Function}

Postoperative liver function and hepatocellular integrity were monitored by routine laboratory parameters.

Statistical Analyses

Calculation of Sample Size

Since hepatic protection by IPC has to be shown by its effects on serum liver enzyme activities, is likely to reflect protection at the metabolic level as well, estimation of sample size was based on the endpoint 'reduction of serum liver enzyme activities'. Assuming that IPC reduces amino alanine and aspartate aminotransferase (ALT, AST) serum enzyme activities to levels of 33\% of the ischemic control group and assuming a first and second type error of 5 and $10 \%$, the number of patients was calculated to 25 per group [5].

Intra- and Intergroup Statistical Comparisons

Demographic data and surgery-related data were normally distributed (one-sample Kolmogorov-Smirnov) and hence tested by ANOVA and post-hoc unpaired t test for detection of intergroup differences. Testing for differences in ratios of females to males between groups was performed by $\chi^{2}$-test. Since the other variables were frequently not normally distributed, parameter-free tests were applied. Multiple comparisons within groups were performed by the Friedman test and post-hoc Wilcoxon test. Comparison of data between groups was done by Kruskal-Wallis test and post-hoc Mann-Whitney U-test.

Since the authors decided not to apply Bonferroni correction despite multiple testing, it should be recognized that this decision could limit the strength of the interpretation and make it necessary to consider marginal findings as completely preliminary. Although type I error could have been reduced by Bonferroni correction, correction by this method would have increased a type II error. Since in the latter case a biological or a clinical relevant effect of IPC that truly exists would not be recognized, our study would fail to allow identification of conditions that are worthwhile analyzing in more detail in future studies. All statistical procedures were two-tailed and a $p$ value $<0.05$ was considered statistically significant. Analyses were performed by SPSS statistical program (SPSS/PC+ Version 10, SPSS Inc., Chicago, Ill., USA).

\section{Results}

\section{Patients' Characteristics and Intraoperative Data}

Demographic data and intraoperative parameters did not significantly differ between patient groups. In particular, there was no difference between groups with respect to the duration of resection, the Pringle maneuver, and the volume of resected liver [16].

\section{Plasma Concentrations of Adenosine and Its \\ Catabolites before and after Preconditioning}

Plasma concentrations of adenosine determined before preconditioning in central venous, arterial and portal venous blood were not significantly different from each other. Early after the start of reperfusion (<90 s), adenosine plasma concentrations increased in portal venous blood but did not reach the level of statistical significance. However, the elevation of adenosine plasma concentrations was paralleled by a more pronounced increase of its metabolites inosine, hypoxanthine and xanthine (fig. 2). 


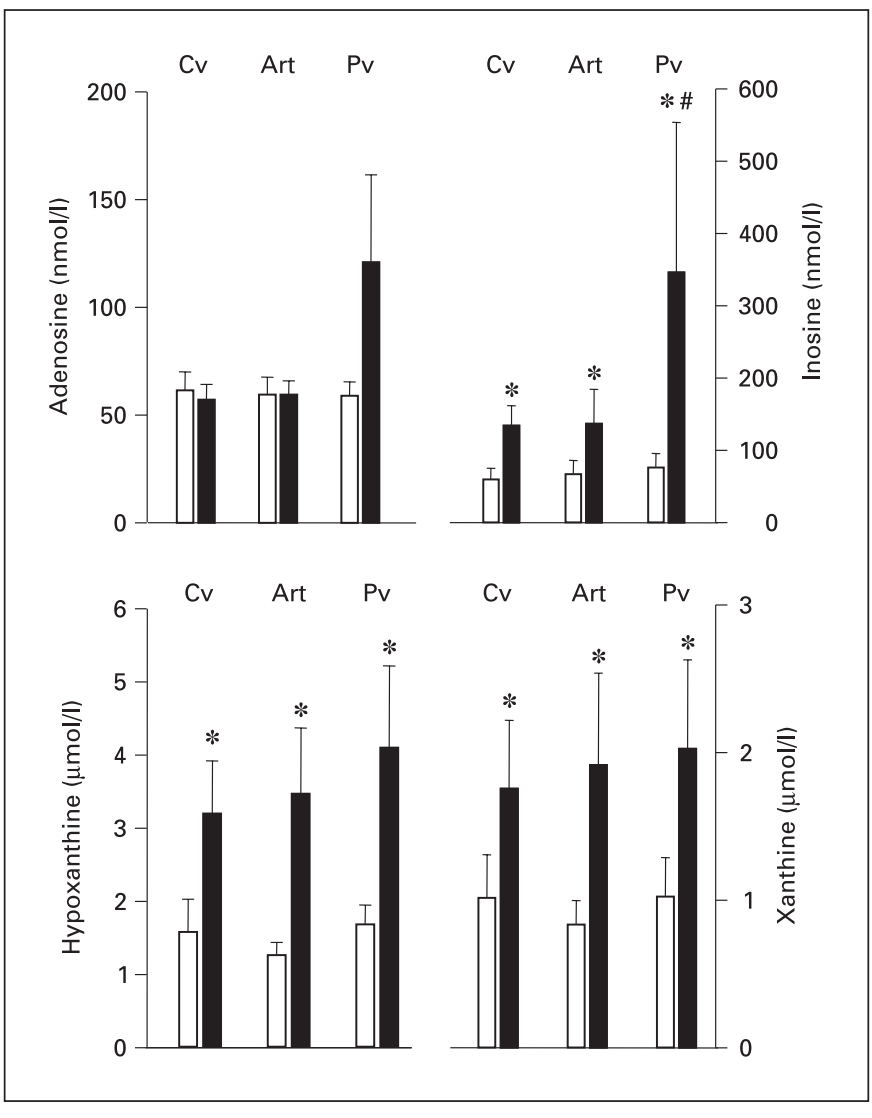

Fig. 2. Central venous $(\mathrm{Cv})$, arterial (Art), and portal venous (Pv) plasma concentrations of adenosine, inosine hypoxanthine and xanthine before $(\square)$ and after ( $\square$ ) IPC. Ischemic preconditioning was performed by $10 \mathrm{~min}$ of portal triad clamping followed by $10 \mathrm{~min}$ of reperfusion. Concentrations shown were determined at the respective sites within the first $90 \mathrm{~s}$ of reperfusion. Means \pm SEM, $\mathrm{n}=21-23, * \mathrm{p}<0.05$ vs. before preconditioning, Wilcoxon test.

\section{Plasma Concentrations of Lactate before and after Preconditioning}

Plasma lactate concentrations increased from $1.10 \pm$ 0.07 to $1.76 \pm 0.10 \mathrm{mmol} / 1$ in central venous blood (means \pm SEM, $\mathrm{n}=25 ; \mathrm{p}<0.05$ ) and from $1.09 \pm 0.07$ to $1.86 \pm 0.11 \mathrm{mmol} / 1$ in portal venous blood (means \pm SEM, $\mathrm{n}=25 ; \mathrm{p}<0.05)$.

\section{Plasma Concentrations of Purines before and after the Pringle Maneuver}

Following $10 \mathrm{~min}$ of ischemia and an additional period of $10 \mathrm{~min}$ of reperfusion, plasma concentrations of inosine, hypoxanthine and xanthine were returned to normal levels (fig. 3 time point 'Pre'). Thus, plasma concentrations of purine nucleosides and bases of patients previously preconditioned were not significantly different from those determined in patients of the other groups. After partial hepatectomy in the absence of the Pringle maneuver (control group I), no significant changes of all three types of plasma purines occurred with the exception of hypoxanthine determined in arterial and portal venous blood.

In contrast to control group I, group II patients who were subjected to the Pringle maneuver during partial hepatectomy exhibited a strong increase of all purines at all sampling sites with the highest values being observed 3 min after start of reperfusion.

The rise of purine plasma concentrations caused by the Pringle maneuver was decreased at all sampling sites in patients who had previously undergone preconditioning (group III). The attenuation of purine plasma concentrations by IPC was most pronounced during early reperfusion (3 and $15 \mathrm{~min}$ ) and was statistically significant different between groups II and III for inosine and hypoxanthine in portal venous blood.

With respect to the plasma concentrations of adenosine, no changes were observed in patients of the control group. In group II patients only a slight rise occurred during early reperfusion. These effects, however, did not reach the level of statistical significance (data not shown).

\section{Calculated Differences: Portal Venous minus Arterial}

\section{Purine Plasma Concentration}

In order to estimate formation of purines in the intestine, purine plasma concentration determined in arterial blood was subtracted from the concentration measured in portal venous blood specimens taken simultaneously. There were no differences between groups prior to the start of the Pringle maneuver or partial hepatectomy with respect to all types of purines studied (table 1). Upon reperfusion the portal venous minus arterial plasma concentration differences became positive for inosine and hypoxanthine only in patients of group II, with the difference for hypoxanthine being significantly lower in patients previously preconditioned (group III).

\section{Plasma Lactate Concentrations}

IPC caused an elevation in plasma lactate concentrations (see also section 'Plasma Concentrations of Lactate Before and After Preconditioning'). Although another 10-min period of reperfusion had passed after IPC until the next blood samples were taken for determination of baseline 'Pre resection' plasma lactate concentrations, there was still a significant difference between groups II 


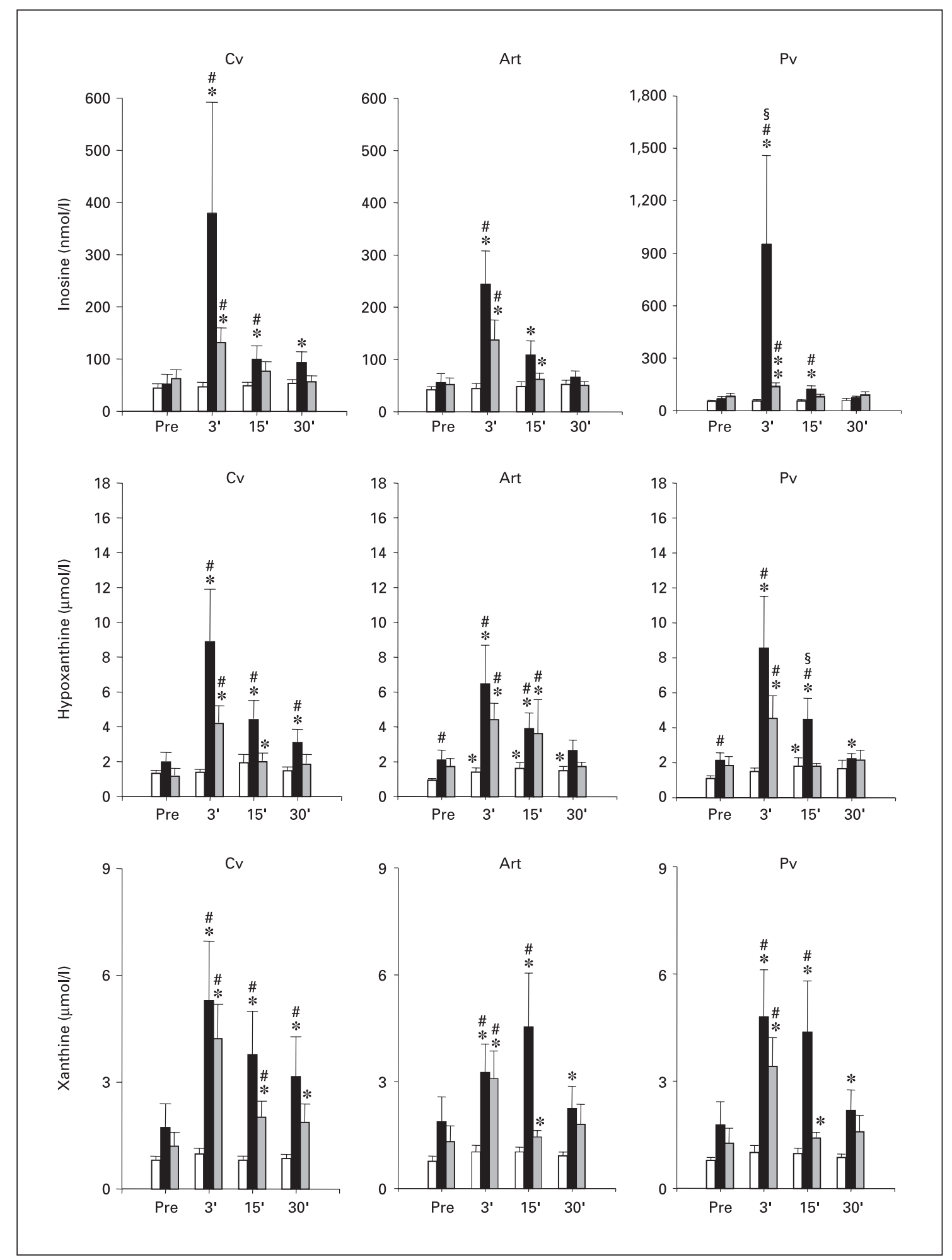

Fig. 3. Central venous $(\mathrm{Cv})$, arterial (Art), and portal venous $(\mathrm{Pv})$ plasma concentrations of inosine, hypoxanthine and xanthine before and after partial hepatectomy without clamping of the portal triad (group I, $\square$ ), with clamping of the portal triad, i.e. Pringle maneuver (group II, $\square$ ) and with the Pringle maneuver preceded by IPC (group III, 网). Pre: before start of the Pringle maneuver or partial hepatectomy. 3', 15', 30': time in minutes after start of reperfusion. Means \pm SEM, $n=16-23, * \mathrm{p}<0.05$ vs. Pre, Friedman and post-hoc Wilcoxon test; ${ }^{\#} \mathrm{p}<0.05$ vs. group I, ${ }^{\S}$ vs. group III, Kruskal-Wallis test and post-hoc Mann-Whitney test. 
Table 1. Portal venous minus arterial plasma concentration differences of inosine, hypoxanthine and xanthine

Table 2. Lactate plasma concentrations $(\mathrm{mmol} / \mathrm{l})$

\begin{tabular}{|c|c|c|c|}
\hline & Group I & Group II & Group III \\
\hline \multicolumn{4}{|l|}{ Inosine, nmol/1 } \\
\hline Pre resection & $7.19 \pm 7.52$ & $8.80 \pm 11.14$ & $28.0 \pm 10.2$ \\
\hline $3 \mathrm{~min}$ after resection/reperfusion & $71.00 \pm 59.0$ & $279.0 \pm 267$ & $5 \pm 22.0$ \\
\hline $15 \mathrm{~min}$ after resection/reperfusion & $4.4 \pm 7.6$ & $10.3 \pm 17.0$ & $16.9 \pm 5.8$ \\
\hline \multicolumn{4}{|l|}{ Hypoxanthine, $\mu \mathrm{mol} / 1$} \\
\hline Pre resection & $0.28 \pm 0.038$ & $-0.03 \pm 0.25$ & $-0.108 \pm 0.13$ \\
\hline $3 \mathrm{~min}$ after resection/reperfusion & $-0.083 \pm 0.11$ & $0.998 \pm 0.36^{\S}$ & $-0.445 \pm 0.5$ \\
\hline $15 \mathrm{~min}$ after resection/reperfusion & $0.244 \pm 0.1$ & $0.291 \pm 54$ & $0.117 \pm 0.16$ \\
\hline \multicolumn{4}{|l|}{ Xanthine, $\mu \mathrm{mol} / \mathrm{l}$} \\
\hline Pre resection & $-0.02 \pm 0.005$ & $-0.07 \pm 0.01$ & $-0.03 \pm 0.009$ \\
\hline $3 \mathrm{~min}$ after resection/reperfusion & $-0.09 \pm 0.053$ & $-0.06 \pm 0.028$ & $-0.157 \pm 0.096$ \\
\hline $15 \mathrm{~min}$ after resection/reperfusion & $-0.04 \pm 0.028$ & $-1.47 \pm 0.78$ & $-0.03 \pm 0.014$ \\
\hline
\end{tabular}

Means \pm SEM, $\mathrm{n}=14-19 .{ }^{\S} \mathrm{p}<0.05$ vs. group III. For further explanations, see legend to figure 2 .

\begin{tabular}{llll}
\hline & Group I & Group II & Group III \\
\hline Lactate (mmol/l) central venous & & & \\
$\quad$ Pre resection & $1.18 \pm 0.11$ & $1.20 \pm 0.11$ & $1.5 \pm 0.10^{\$}$ \\
3 min & $1.43 \pm 0.17^{*}$ & $2.47 \pm 0.22^{*, \#}$ & $2.33 \pm 0.14^{*, \#}$ \\
15 min & $1.38 \pm 0.17^{*}$ & $2.27 \pm 0.23^{*, \#}$ & $1.97 \pm 0.16^{*, \#}$ \\
30 min & $1.35 \pm 0.20^{*}$ & $2.12 \pm 0.22^{*}$ & $1.95 \pm 0.17^{*, \#}$ \\
2 h & $1.65 \pm 0.166^{*}$ & $2.33 \pm 0.28^{*, \#}$ & $1.97 \pm 0.16^{*, \#}$ \\
3 min minus Pre values & $0.29 \pm 0.10$ & $1.27 \pm 0.17^{\#}$ & $0.82 \pm 0.11^{*, \$}$ \\
\hline Lactate (mmol/l) portal venous & & & \\
Pre resection & $1.14 \pm 0.11$ & $1.20 \pm 0.11$ & $1.44 \pm 0.10$ \\
3 min & $1.52 \pm 0.18^{*}$ & $2.68 \pm 0.27^{*, \#}$ & $2.27 \pm 0.14^{*, \#}$ \\
15 min & $1.38 \pm 0.19^{*}$ & $2.17 \pm 0.24^{*, \#}$ & $1.97 \pm 0.17^{*, \#}$ \\
30 min & $1.41 \pm 0.13^{*}$ & $2.14 \pm 0.27^{*, \#}$ & $1.88 \pm 0.22^{*, \#}$ \\
3 min minus Pre values & $0.34 \pm 0.12$ & $1.54 \pm 0.24^{\#}$ & $0.86 \pm 0.13^{\#, \$}$ \\
\hline
\end{tabular}

Means \pm SEM, $\mathrm{n}=25, * \mathrm{p}<0.05$ vs. Pre resection or Pringle, Friedman and post-hoc Wilcoxon test; ${ }^{\#} \mathrm{p}<0.05$ vs. group I, ${ }^{\$} \mathrm{p}<0.05$ vs. group II, Kruskal-Wallis and post-hoc Mann-Whitney test.

and III in central venous lactate concentrations prior to the start of the Pringle maneuver (see table 2: 'Pre resection'). After the end of the Pringle maneuver, plasma concentrations of lactate increased further in both groups. The values determined during reperfusion were reduced by IPC but were not statistically significant. However, when the absolute rise in the plasma concentrations between Pre resection and 3 min after the Pringle maneuver was calculated (see table 2: 3 min minus Pre values) and compared between both groups, the absolute concentration changes determined in central venous and portal venous blood were significantly diminished by IPC.

\section{Parameters of Hepatocellular Injury and Function}

No preoperative significant differences in serum activities (IU) were observed between groups with respect to alanine transferase (ALT: range 21.5-32.7) or aspartate transferase (AST: range 15.0-22.7). When the Pringle maneuver was applied (group II), ALT and AST were significantly $(\mathrm{p}<0.05)$ elevated as compared with the non-Pringle group (group I) on the first and second post- 
operative days (POD1 and POD2) (POD1: ALT: group I: $254.3 \pm 62.4$ vs. group II: $519.4 \pm 91$, and AST: group I: $166 \pm 27$ vs. group II: $420 \pm 104$; POD2: ALT: group I: $195.8 \pm 21$ vs. group II: $497 \pm 145$ and AST: group I: $88 \pm 21$ vs. group II: $246 \pm 67$ ). When IPC was applied (group III), the Pringle maneuver-induced rise of serum ALT and AST activities was significantly attenuated only for ALT on the second postoperative day as compared with group II (group III: ALT: POD1: $261.4 \pm$ 40.9; POD 2: $208.5 \pm 36.5$ ( $\mathrm{p}<0.05$ vs. group II) and AST: POD 1: $226.4 \pm 3.4$; POD 2: $105 \pm 16$ ).

In comparison to control group I, the Pringle maneuver also significantly increased postoperative total serum bilirubin concentrations and decreased thromboplastin times and serum factor $\mathrm{V}$ activities. These Pringle maneuver-associated disturbances in liver secretory function and protein synthesis, however, were not significantly attenuated by IPC (data not shown).

\section{Discussion}

\section{Role of Adenosine in Hepatic Preconditioning}

Ischemia has for a long time been known to cause the degradation of adenine nucleotides leading to the enhanced formation of adenosine and its metabolites inosine, hypoxanthine and xanthine. More recently, formation of adenosine during ischemia has regained attention, because this nucleoside is suggested to be a central mediator of protection in IPC [9]. With respect to the liver, adenosine has been shown in many animal models to protect from tissue injury induced by cold [2] as well as warm ischemia [1]. Specifically, modulation of tissue adenosine by exogenous application [1] or by increasing its endogenous concentrations [10] were shown to be as protective as IPC to prevent from ischemia-reperfusion injury. Moreover, activation or antagonism of $\mathrm{A}_{2}$ receptors by specific agonists [11] and antagonists [3] mimicked or abrogated the protection of the liver afforded by IPC, respectively. In humans, recent studies provided also good evidence for adenosine to exert some of the protective effects of IPC, although these observations were limited to the heart $[12,13]$. There is also increasing evidence for protective effects of IPC in major liver resection as suggested by significantly reduced hepatocellular injury [14-16] and improved intraoperative hemodynamic stability. Additionally, IPC not only allowed to attenuate ischemic liver injury but to also take advantage of the Pringle maneuver to reduce blood loss during resection by up to $\sim 65 \%$ [15]. However, the role of adeno- sine in hepatic preconditioning was not considered so far. Thus, in the present study we tested (i) whether adenosine plasma concentrations were transiently increased by IPC and (ii) whether the Pringle maneuver-induced elevations of plasma purines could be attenuated by IPC.

As shown in figure 2, plasma concentrations of adenosine were increased during the early phase of reperfusion in portal venous blood. However, this rise in adenosine was not significant statistically but was paralleled by significant increases of its major metabolites inosine, hypoxanthine and xanthine, indicating that substantial formation of adenosine had taken place (fig. 1, 2).

Enhanced formation of adenosine's degradation products due to hepatic preconditioning is in agreement with depletion of ATP, adenosine diphosphate (ADP), adenosine monophosphate (AMP) and accumulation of adenosine, inosine, hypoxanthine, and xanthine within 10 min of warm liver ischemia $[11,17,18]$. Of course, the demonstration of a significant rise in portal venous plasma concentrations of adenosine's degradation products is no definite proof for adenosine being a central mediator in hepatic preconditioning. To this end, pharmacologic preconditioning of the human liver by transient intravenous infusion of adenosine would appear to be a more appropriate tool to address this question under clinical conditions.

\section{Effects of IPC on Plasma Metabolites Reflecting \\ Severity of Tissue Ischemia}

Currently, there is little doubt on the protective potency of IPC for the liver. In many animal models of warm liver injury, ischemic preconditiong was not only effective to attenuate cell damage but also to better preserve those cell functions known to be dependent on the availability of ATP. Among these, secretion of bile is a process which is quite sensitive to a lack in cellular energy. In fact, in animal models, IPC improved post-ischemic bile excretion [19]. Moreover, several investigators demonstrated that ischemic or pharmacologic preconditioning (use of $\mathrm{A}_{2}$ receptor agonists) decreases the loss in tissue levels of ATP and ADP during a subsequent more severe ischemic period $[11,18]$. In these studies, the IPCmediated preservation of adenine nucleotides was also reflected by a reduction of inosine, hypoxanthine and xanthine accumulation, respectively [18]. Formation of lactate was also diminished. Similar observations have been reported from animal models of myocardial preconditioning, suggesting tissue protection by preconditioning through preservation of ATP stores and reducing lactate accumulation [20]. In agreement with these observations 
in animal models, patients subjected to IPC showed lower plasma concentrations of inosine, hypoxanthine and xanthine after the Pringle maneuver than patients without preconditioning (fig. 3). In parallel, the rise in the plasma concentrations of lactate was also attenuated by IPC (table 2). However, significant reduction of purines by IPC could be only observed in portal venous blood for inosine and hypoxanthine. In consideration of the less pronounced effects detected in central venous blood, it should be taken into account that relatively high concentrations of purines leaving the liver during early reperfusion were likely to be diluted with purine-poor venous blood returning from the lower and upper caval vein before being collected from the central venous line. Despite these limitations, amelioration of plasma purine metabolites and lactate in preconditioned patients may yet indicate preservation of cellular energy homeostasis as already shown in various animal models $[11,18,20]$. In these studies, more detailed analyses of biochemical pathways led to the conclusion of induction of 'metabolic arrest' in terms of less ATP depletion and lactate accumulation as a possible mechanism by which IPC protects tissues during ischemia [18]. Very recently, Clavien et al. [21] published a prospective randomized clinical study in which they confirmed their own previously described protective effect of IPC in patients undergoing major liver resection under inflow occlusion. Although adenine nucleotides were analyzed in liver tissue samples that were not taken by in situ freeze clampage, Clavien et al. showed exemplarily with 5 patients per group a statistically significant higher post-ischemic hepatic ATP content in patients that were pretreated by IPC.

\section{Role of the Intestine in Hepatic Preconditioning}

Because we were not able for ethical and technical reasons to perform direct measurements of tissue concentrations of purines and lactate, we determined plasma concentrations of energy-dependent metabolites at different sampling sites in order to calculate concentration differences across vascular beds. Surprisingly, portal venous concentrations of purines significantly increased in response to the Pringle maneuver. Even more important, this increase was significantly attenuated by IPC. Moreover, when the difference between plasma concentrations of purines between portal venous blood and arterial blood was calculated, a positive difference was obtained for patients who underwent the Pringle maneuver without preconditioning (table 1). In contrast, in patients with preconditioning, the corresponding calculated difference leveled around zero. These results suggest that clamping of the portal triad, i.e. occlusion of the venous outflow of the intestine, causes severe congestion of the nutritive blood flow which leads to the net production of purine metabolites. Thus, not only the liver but also the intestine was obviously subjected to compromises of its energy metabolism by the Pringle maneuver and the associated net production of purines in the intestine could be prevented by IPC. IPC might, hence, protect not only the liver but also the intestine from ischemic injury. To further substantiate this conclusion, one wishes to have determined tissue concentrations of purines or other parameters of intestine lesions. Taking biopsies of the intestinal wall, however, represents an unacceptable risk for severe intraabdominal infectious complications due to possible secondary bowel perforation.

\section{Clinical Model and Efficacy of IPC}

In the present study, patients were enrolled in a prospective manner and duration of the Pringle maneuver was adapted to surgical requirements. Accordingly, the results were obtained under clinical conditions of routine surgery with unavoidable variations of ischemia (Pringle) times. Despite the heterogeneity of the duration of the Pringle maneuver, IPC appeared to be effective in attenuating hepatocellular injury as assessed by the postoperative serum liver transferase activities (see Results and Choukèr et al. [16]).

In conclusion, the results of this prospectively randomized clinical study demonstrate that IPC very likely enhances formation of adenosine as reflected by increased central and portal venous plasma concentrations of adenosine's degradation products inosine, hypoxanthine and xanthine. In addition, IPC attenuates disturbances in the hepatic energy metabolism caused by a subsequent more severe ischemic period as suggested by the decreased plasma concentrations of purines following the Pringle maneuver. In agreement with results from animal models suggesting a 'metabolic arrest', IPC diminished plasma lactate concentrations during reperfusion in humans as well. Most interestingly, IPC also decreased formation of purines in the intestine. Thus, protection of the human liver from ischemic tissue injury may not only be afforded by direct adaptive metabolic effects within the liver, but might result also from preconditioning of the intestine. 


\section{Acknowledgments}

The authors gratefully acknowledge the skillful technical assistance of Stefan Meindl, Marion Hörl and Gaby Kröger from the Department of Anesthesiology. The authors would like to thank Prof. Dr. Boos from the Department of Clinical Chemistry, Klini- kum Grosshadern, LMU, Munich, for providing internal surface nitrophenylboronic acid precolumns. We would also like to thank Charles Caldwell for critical reading of the manuscript. Some of this research was conducted by Andre Martignoni in partial fulfillment of the requirements for a doctorial thesis from the Medical Faculty of Ludwig Maximilians University of Munich, Germany.

\section{References}

1 Peralta C, Hotter G, Closa D, Gelpi E, Bulbena O, Rosello Catafau J: Protective effect of preconditioning on the injury associated to hepatic ischemia-reperfusion in the rat: Role of nitric oxide and adenosine. Hepatology 1997;25: 934-937.

2 Arai M, Thurman RG, Lemasters JJ: Contribution of adenosine $\mathrm{A}_{2}$ receptors and cyclic adenosine monophosphate to protective ischemic preconditioning of sinusoidal endothelial cells against storage/reperfusion injury in rat livers. Hepatology 2000;32:297-302.

3 Peralta C, Hotter G, Closa, Pratts N, Xaus C, Gelpi E, Rosello Catafau J: The protective role of adenosine in inducing nitric oxide synthesis in rat liver ischemia preconditioning is mediated by activation of adenosine $\mathrm{A}_{2}$ receptors. Hepatology 1999;29:126-132.

4 Moser GH, Schrader J, Deussen A: Turnover of adenosine in plasma of human and dog blood. Am J Physiol 1989;256:C799-C806.

5 Pocock SJ: The size of a clinical trial; in Stuart SJ (ed): Clinical Trials. A Practical Approach. Chichester, Wiley, 1996, pp 123-141.

6 Rau HG, Schardey HM, Buttler E, Reuter C, Cohnert TU, Schildberg FW: A comparison of different techniques for liver resection: Blunt dissection, ultrasonic aspirator and jet-cutter. Eur J Surg Oncol 1995;21:183-187.

7 Hagemeier E, Kemper K, Boos KS, Schlimme E: On-line high-performance liquid affinity chromatography-high-performance liquid chromatography analysis of monomeric ribonucleoside compounds in biological fluids. $\mathrm{J}$ Chromatogr 1983;282:663-669.
8 Ontyd J, Schrader J: Measurement of adenosine, inosine, and hypoxanthine in human plasma. J Chromatogr 1984;307:404-409.

9 De Jong JW, de Jonge R, Keijzer E, Bradamante $\mathrm{S}$ : The role of adenosine in preconditioning. Pharmacol Ther 2000;87:141-149.

10 Howell JG, Zibari GB, Brown MF, Burney DL, Sawaya DE, Olinde JG, Granger DN, McDonald JC: Both ischemic and pharmacological preconditioning decrease hepatic leukocyte/ endothelial cell interactions. Transplantation 2000;69:300-303.

11 Nakayama H, Yamamoto Y, Kume M, Yamagami K, Yamamoto H, Kimoto S, Ishikawa, Ozaki N, Shimahara Y, Yamaoka Y: Pharmacologic stimulation of adenosine $\mathrm{A}_{2}$ receptor supplants ischemic preconditioning in providing ischemic tolerance in rat livers. Surgery 1999; 126:945-954.

12 Hawaleshka A, Jacobsohn E: Ischaemic preconditioning: Mechanisms and potential clinical applications. Can J Anaesth 1998;45:670682.

13 Alkhulaifi AM: Preconditioning the human heart. Ann R Coll Surg Engl 1997;79:49-54.

14 Clavien PA, Yadav S, Sindram D, Bentley RC: Protective effects of ischemic preconditioning for liver resection performed under inflow occlusion in humans. Ann Surg 2000;232:155162.

15 Choukèr A, Schachtner T, Schauer R, Dugas M, Löhe F, Martignoni A, Pollwein B, Niklas M, Rau HG, Jauch KW, Peter K, Thiel M: Effects of Pringle manoeuvre and ischaemic preconditioning on haemodynamic stability in patients undergoing elective hepatectomy: A randomised trial. Br J Anaesth 2004;93:204211.
16 Choukèr A, Martignoni A, Schauer RJ, Dugas M, Rau HG, Jauch KW, Peter K, Thiel M: Beneficial effects of ischemic preconditioning in patients undergoing hepatectomy: The role of neutrophils. Arch Surg 2005;140:129-136.

17 Peralta C, Closa D, Xaus C, Gelpi E, Rosello Catafau J, Hotter G: Hepatic preconditioning in rats is defined by a balance of adenosine and xanthine. Hepatology 1998;28:768-773.

18 Peralta C, Bartons R, Riera L, Manzano A, Xaus C, Gelpi E, Rosello Catafau J: Hepatic preconditioning preserves energy metabolism during sustained ischemia. Am J Physiol 2000; 279:G163-G171.

19 Yoshizumi T, Yanaga K, Soejima Y, Maeda T, Uchiyama H, Sugimachi K: Amelioration of liver injury by ischaemic preconditioning. $\mathrm{Br} \mathrm{J}$ Surg 1998;85:1636-1640.

20 Murry CE, Richard VJ, Reimer KA, Jennings $\mathrm{RB}$ : Ischemic preconditioning slows energy metabolism and delays ultrastructural damage during a sustained ischemic episode. Circ Res 1990;66:913-931.

21 Clavien PA, Selzner M, Rudiger HA, Graf R, Kadry Z, Rousson V, Jochum W: A prospective randomized study in 100 consecutive patients undergoing major liver resection with versus without ischemic preconditioning. Ann Surg 2003;238:843-850.

22 Sitkovsky MV, Lukashev D, Apasov S, Kojima H, Koshiba M, Caldwell C, Ohta A, Thiel M: Physiological control of immune response and inflammatory tissue damage by hypoxia-inducible factors and adenosine $\mathrm{A}_{2} \mathrm{~A}$ receptors. Annu Rev Immunol 2004;22:657-682. 